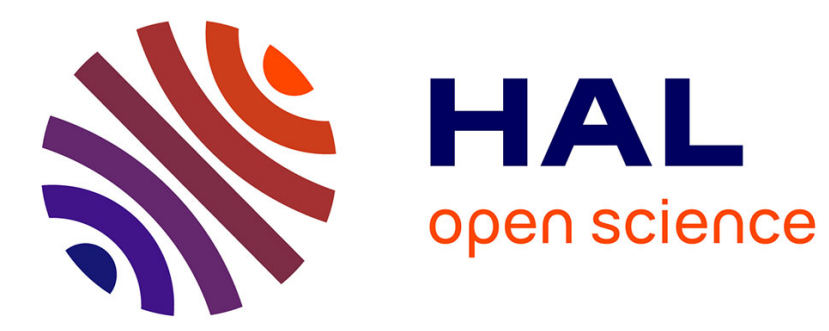

\title{
Chiral colloidal clusters
}

\author{
Djamal Zerrouki, Jean Baudry, David Pine, Paul Chaikin, Jérôme Bibette
}

\section{To cite this version:}

Djamal Zerrouki, Jean Baudry, David Pine, Paul Chaikin, Jérôme Bibette. Chiral colloidal clusters. Nature, 2008, 455 (7211), pp.380-382. 10.1038/nature07237 . hal-01620847

\section{HAL Id: hal-01620847 https://hal.science/hal-01620847}

Submitted on 21 Oct 2017

HAL is a multi-disciplinary open access archive for the deposit and dissemination of scientific research documents, whether they are published or not. The documents may come from teaching and research institutions in France or abroad, or from public or private research centers.
L'archive ouverte pluridisciplinaire HAL, est destinée au dépôt et à la diffusion de documents scientifiques de niveau recherche, publiés ou non, émanant des établissements d'enseignement et de recherche français ou étrangers, des laboratoires publics ou privés. 


\title{
Chiral colloidal clusters
}

\author{
D. Zerrouki ${ }^{1}$, J. Baudry ${ }^{1}$, D. Pine ${ }^{2}$, P. Chaikin ${ }^{2} \&$ J. Bibette ${ }^{1}$
}

Chirality is an important element of biology, chemistry and physics. Once symmetry is broken and a handedness is established, biochemical pathways are set. In DNA, the double helix arises from the existence of two competing length scales, one set by the distance between monomers in the sugar backbone, and the other set by the stacking of the base pairs ${ }^{1}$. Here we use a colloidal system to explore a simple forcing route to chiral structures. To do so we have designed magnetic colloids that, depending on both their shape and induced magnetization, self-assemble with controlled helicity. We model the two length scales with asymmetric colloidal dumbbells linked by a magnetic belt at their waist. In the presence of a magnetic field the belts assemble into a chain and the steric constraints imposed by the asymmetric spheres force the chain to coil. We show that if the size ratio between the spheres is large enough, a single helicity is adopted, right or left. The realization of chiral colloidal clusters opens up a new link between colloidal science and chemistry. These colloidal clusters may also find use as mesopolymers, as optical and light-activated structures ${ }^{2}$, and as models for enantiomeric separation.

To advance the use of colloids in nanotechnology as a route for the fabrication of large-scale complex and useful structures, we need to give the building blocks the functionality and variability found in chemistry, allowing the synthesis of polymers and chiral structures rather than the crystalline structures or aggregates traditionally observed in colloids ${ }^{3-7}$. Using colloids to mimic atoms, as building blocks for molecules or macromolecules at a colloidal scale, would also offer insight in designing and rationalizing functionality in chemistry. Steric hindrance between substituents is broadly accepted to be one of the main driving forces directing the formation of a particular configurational isomer ${ }^{8}$. At a colloidal scale, these steric interactions are much easier to control without the confounding influence of hydrogen bonding, van der Waals and electrostatic forces. But although isotropic particles suffice to form amorphous aggregates, crystals or geometrically confined structures ${ }^{9}$, the creation of colloidal molecules requires that the building blocks assemble in a more precise way in order to mimic bond angles. An effective processing route is to apply an external magnetic field to colloids that contain super-paramagnetic material. This way, very long polymeric assemblies can be created ${ }^{10}$. This strategy leads to very rapid assembly, but it has been limited to one-dimensional and uniform structures, thus excluding the formation of configurational isomers.

Here we introduce new magnetic colloids that can rapidly selfassemble into a variety of isomeric forms. Depending on the shape of the colloidal blocks relative to their spontaneous direction of magnetization, these colloids assemble into structures that must fulfil both steric and magnetic constraints. This induces a fixed repeated isomeric configuration or tacticity. We present two types of colloidal assemblies which led us to a third structure- - a helix. The first grows in-plane with a bond angle of $60^{\circ}$ producing a perfect alternation of colloidal block positions, a syndiotactic arrangement. The second grows with a repeating in-plane/out-of-plane structure with a $90^{\circ}$ rotation angle between two blocks. The third is our helical structure.

Individual colloidal blocks are produced using emulsion confinement ${ }^{11}$. We start with droplets containing a binary colloidal mixture comprising both an oil-based ferrofluid and hydrophobic micrometre-sized silica particles. By adjusting the hydrophobicity of the silica particles and the quantity of iron oxide nanoparticles, we can design a specific magnetic patterning of the silica spheres during solvent evaporation. Under different conditions, we obtain four distinct types of colloidal structures after oil evaporation. Scanning electron microscope pictures of these new colloids are shown in Fig. 1. The detailed experimental protocol is described in the Methods. When no silica particles are trapped, we obtain conventional spherical magnetic colloidal particles (Fig. 1a). When only one silica particle is trapped and when both its initial size and ferrofluid content are appropriately set, we obtain silica particles that possess a single characteristic magnetic 'cap' (Fig. 1b). This magnetic cap consists of densely packed $\mathrm{Fe}_{2} \mathrm{O}_{3}$ nanoparticles about $10 \mathrm{~nm}$ in diameter. The de-wetting of the ferrofluid on the curved hydrophobic silica surface during the extraction of the oil drives its shape. At the very end of the evaporation, the nanoparticles irreversibly stick together and adhere to the silica surface, leading to the final structure. When two particles are trapped within one emulsion droplet, we obtain doublets of silica particles with a solid magnetic ring located around the contact point between the two silica spheres. This ring is shaped both by the silica contour and by capillary forces confining the ferrofluid during oil evaporation. We refer to these magnetic structures as 'rings' and the binary particles as 'dumbbells'. We have made these dumbbells with either the same or differently sized silica particles, as shown in Fig. 1c and d.

Paramagnetic spherical particles as in Fig. 1a do not individually orient in a magnetic field, but two particles will attract and align in the field direction owing to shape anisotropy (part a, Fig. 2A, B). The magnetic caps and rings on the silica colloids are not magnetically isotropic and their shape anisotropy causes them to align individually in an applied field with their circular base parallel to the field ${ }^{12}$. Two such particles will attract but their steric interaction will cause the pair not only to align with the field but to adopt specific configurations relative to each other. The capped particles have a magnetic dipole interaction which is maximized when the circular bases of the caps are parallel to each other, parallel to the field and as close together as possible. The capped particles can align with their caps on the same or opposite sides of the particles. In the latter arrangement the caps are closer (part b, Fig. 2A, B). On the symmetric dumbbells the rings form a small 'belt' around the waist of the touching spheres ( $\left.r_{\text {ring }} \ll r_{\text {sphere }}\right)$. The attractive $1 / r^{3}$ interaction is largest when the belts are as close as possible along the field. This occurs when the dumbbells are perpendicular to the magnetic field and to each other (part c, Fig. 2A, B). Two asymmetric dumbbells also adopt a 'closest belt' approach with all spheres touching (part d, Fig. 2A, B). 


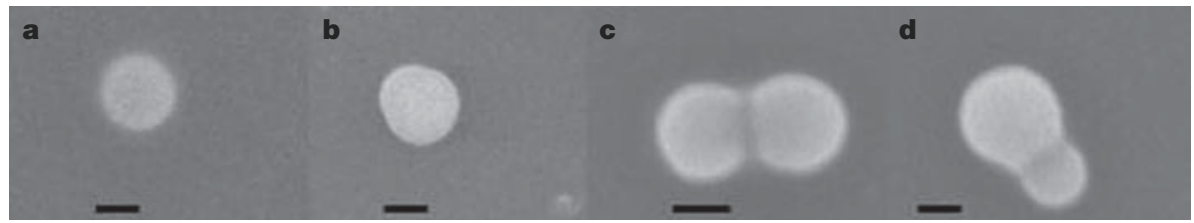

Figure 1 | Electron microscopy images of particles showing magnetic patterning. Typical scanning electron microscope pictures of dried alcanebased ferrofluid droplets. a, Ferrofluid only; the ferrofluid nanoparticles collapse into a spherical colloidal particle. b, Ferrofluid with one silica particle of diameter $1 \mu \mathrm{m}$; the particle is decorated by a magnetic pattern consisting of nanoparticles of $\mathrm{Fe}_{2} \mathrm{O}_{3}$. The cap is clearly visible when the

In Fig. 3 we show our four different colloidal samples under a magnetic field. In all cases the attractive induced dipole-dipole interaction is sufficient to drive the self-assembly. In Fig. 3a, isotropic spherical particles form linear chains, as expected. In Fig. 3b, following the steric repulsion that we have seen in pairs in part b of Fig. 2A, B, 'caps' order alternately up and down along the chain direction leading to isomeric tacticity at the colloidal scale. Indeed, this assembly is reminiscent of a syndiotactic arrangement observed in linear polymeric chains, for which steric hindrance of substituents drives the regular alternation of particles ${ }^{13}$. For sufficiently thin caps the structure adopts a planar configuration with a bond angle of about $60^{\circ}$ and a torsional and bond rigidity that depends on field strength. Because of the colloidal scale, the dynamics of this mode can be directly recorded in real time using a microscope and video camera (see Supplementary Movie 1). Symmetric dumbbells (Fig. 3c) self-assemble with the neighbouring dumbbells perpendicular to each other and to the field direction. Just as for chains of caps, at lower fields, thermally induced torsional modes can be excited and directly recorded in real time with a microscope (see Supplementary Movie 2).

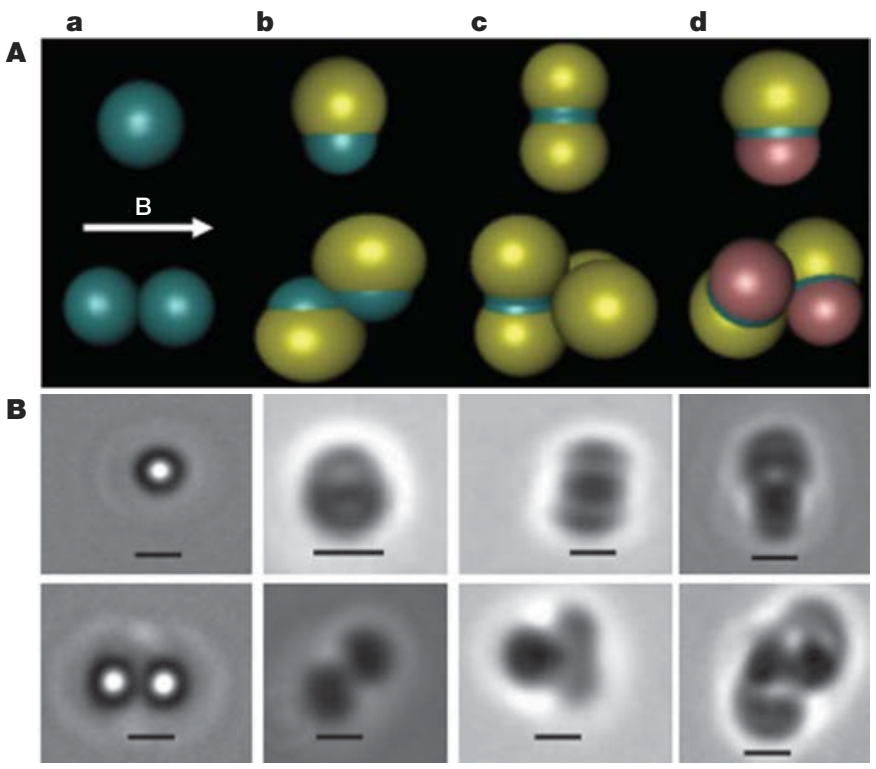

Figure 2 | Competition between magnetic interactions and steric hindrance. A, Schematic diagrams of the magnetic and steric pair interaction between colloidal blocks of types a to d. a, One isotropic paramagnetic particle has no preferential orientation in the field B; two of them attract to form a doublet oriented in the field direction. $b$, One capped particle orients such that the cap base is parallel to the field; two of them adjust their position so that the bases align and touch each other, forcing this up-and-down configuration. c, One symmetric dumbbell orients in the field so that the ring plane becomes parallel to the field; two of them must then rotate by $90^{\circ}$ relative to each other such that the ring planes are as close as possible while retaining the right orientation. $d$, The same argument holds for a pair of asymmetric dumbbells. B, Microscopic pictures of single and paired building blocks of each type, following the same ordering as in A. Magnetic field, $10 \mathrm{mT}$; scale bars, $1 \mu \mathrm{m}$. particle is oriented as shown here. c, Ferrofluid with two identical silica particles of diameter $1 \mu \mathrm{m}$. In this case the magnetic part consists of a ring in between the two silica spheres. d, Ferrofluid with different particle sizes: the silica particles are 1 and $2 \mu \mathrm{m}$ in diameter. Owing to the purification procedure we systematically find about $10 \%$ of other types of clusters in the final samples. Scale bars, $1 \mu \mathrm{m}$.

We now come to the point of emergence of chirality. It is known that monodisperse spheres interacting through excluded volume interactions and confined within a proper geometry can pack with a helical structure ${ }^{9}$. Here we aim at demonstrating what could be the simplest building blocks for self-assembling mesoscopic helical structures in the absence of confinement constraints. First note that in contrast to the symmetric dumbbells of Fig. 3c, a pair of magnetically linked asymmetric dumbbells, the case shown in Fig. $3 \mathrm{~d}$ is in principle already chiral: mirror images do not superimpose, owing to the presence of the rings. Indeed, if we look at the tetrahedron formed by the spheres alone, there are two orthogonal mirror planes, but the reflection symmetry is broken by the magnetic rings (part a, Fig. 4A). A reflection plane can be restored if a third dumbbell is added symmetrically (part b, Fig. 4A). However, a more favourable magnetic interaction, with a more collinear alignment of the rings, occurs if we flip the last dumbbell (part c, Fig. 4A). The combination of a more collinear magnetic interaction plus steric repulsion favours the helical structure when the ratio of the spheres is within a critical range of values, which our calculations show to be from about 2 to 3 . The calculations include the dipole-dipole interactions between rings as well as the hard sphere interactions between different spheres, but neglect the role of temperature, which must decrease the lower limit. Our first choice of a ratio of 2 for our dumbbells was based on this calculation. The movie frames in Fig. 4B show how a single dumbbell approaches a chiral cluster, then rotates and links

a
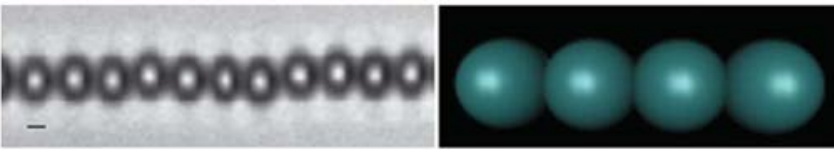

b
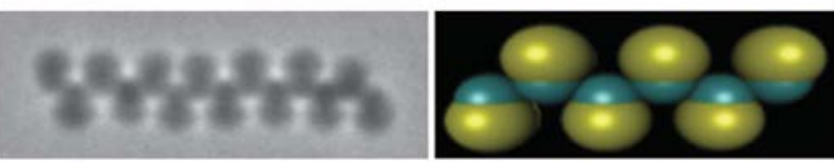

c

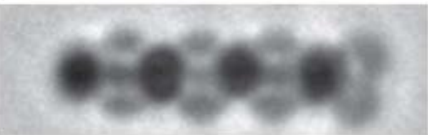

d
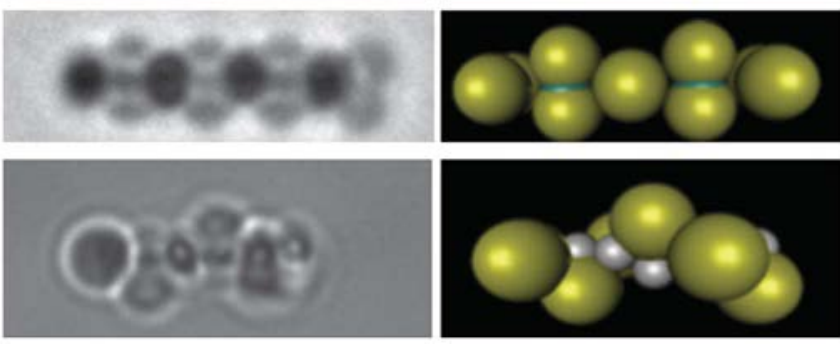

Figure 3 | Self-assembly of blocks into chains under a magnetic field. a-d, Optical microscope images and their corresponding schematic representations. a, Isotropic particles self-assemble into linear chains. b, Caps self-assemble by ordering alternatively up and down along the chain direction. c, To satisfy both steric and magnetic constraints, symmetric dumbbells must rotate by $90^{\circ}$ relative to their neighbours along the chain and field direction. d, Asymmetric dumbbells self-assemble into a helical structure, as a result of the steric hindrance induced by the size difference. Magnetic field, $10 \mathrm{mT}$; scale bars, $1 \mu \mathrm{m}$. 
A
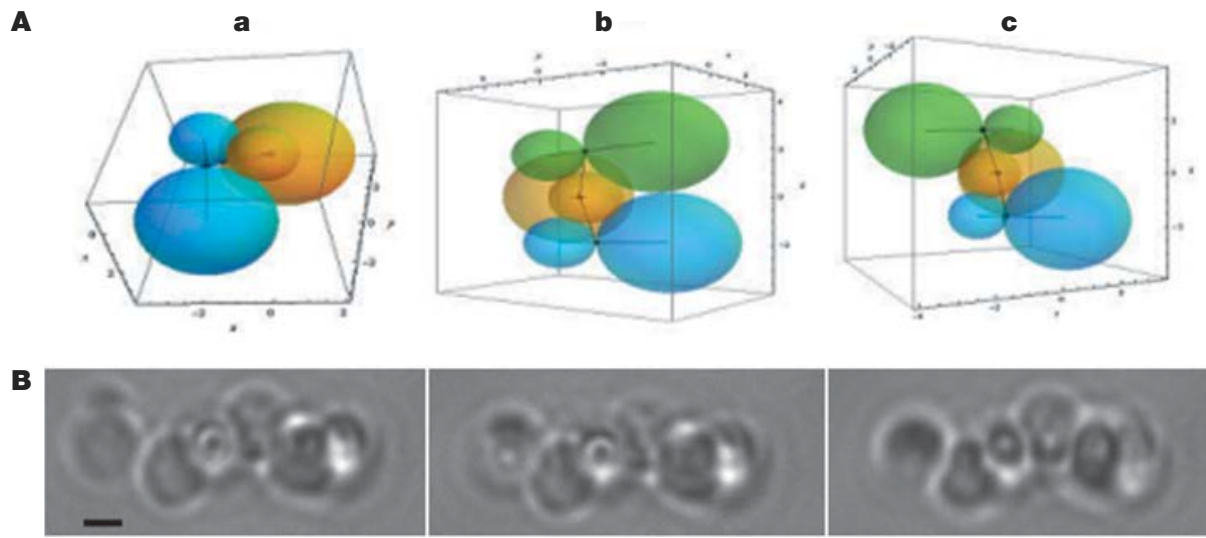

Figure 4 | Configurations and kinetic pathway for dumbbell assembly. A, Schematics showing a pair of dumbbells (a), and the two ways in which a third dumbell could be added to that pair (b and c). B, Optical microscopic

into the correct helical configuration. Of course, the kinetic pathways are complex and require further investigation, including the role of entropy and hydrodynamics. Nevertheless, we emphasize that in spite of the remaining questions about kinetic pathways and the related distributions of chiral isomers, the configurational energy is lower for helical stacking when the ratio of sphere sizes is within the critical range 2 to 3 , and provides insight into the helix formation we observe.

These experiments demonstrate that colloids can build interesting conformational isomers. By controlling the shape and interactions between colloidal building blocks, a variety of structures can be designed. Here we have restricted our efforts to the growth of syndiotactic and chiral structures. We believe, however, that this work opens new routes to produce and self-assemble a wide variety of structures based on the same principles. Moreover, we believe this work could offer some guidance to the assembly of nanoparticles, which has interesting optical applications.

\section{METHODS SUMMARY}

Hydrophilic silica colloids are synthesized by hydrolysis of tetraethylorthosilicate in aqueous solution containing ethanol and ammonia, using a modified seeded growth procedure originally described by Stober and Fink ${ }^{14}$. The silica particles are surface-coated with octyldimethylchlorosilane to redisperse them into octane-based ferrofluid. This mixture is then gently emulsified into an aqueous phase containing Pluronic F127 surfactant and polyvinylpyrolidone. The emulsion is further transformed under controlled shear into a quasi-monodisperse emulsion with globules of diameter $\sim 3 \mu \mathrm{m}$ (ref. 15). The final diluted emulsion is treated with a Rotary Evaporator at $80^{\circ} \mathrm{C}$ under reduced pressure for $2 \mathrm{~h}$ to evaporate the octane that remains in the globules. Cluster separation is obtained with a column that has a density gradient established by stacking solutions of various sucrose concentrations in deionized water. After $24 \mathrm{~h}$ of sedimentation, bands are observed and well separated such that they can be pumped out with a syringe; each fraction is then magnetically sedimented and washed several times. The colloids are examined with an optical microscope equipped with a controllable magnetic field induced by coils. For each type of building block a $50-\mu \mathrm{m}$-thick cell is filled with a solution with a $0.01 \%$ volume fraction of colloidal blocks and allowed to sediment for $1 \mathrm{~min}$. For blocks as in parts a, b and c of Fig. 2A, a homogeneous magnetic field of $10 \mathrm{mT}$ is applied. Blocks self-assemble instantly. For blocks as in part d (asymmetric dumbbells), a field of $10 \mathrm{mT}$ is gradually applied over $1 \mathrm{~min}$. This ensures that a larger proportion of dumbbells assemble within the lower-magnetic-energy configuration in order to nucleate helices. Colloids in their dried state are imaged with a scanning electron microscope in secondary electron mode after sputter-coating with gold.

1. Watson, J. D. \& Crick, F. H. C. A structure for deoxyribose nucleic acid. Nature 171, 737-738 (1953).

2. Dreyfus, R. et al. Microscopic artificial swimmers. Nature 437, 862-865 (2005).

3. Leunissen, M. E. et al. Ionic colloidal crystals of oppositely charged particles. Nature 437, 235-240 (2005).

4. Savage, J. R., Blair, D. W., Levine, A. J., Guyer, R. A. \& Dinsmore, A. D. Imaging the sublimation dynamics of colloidal crystallites. Science 314, 795-798 (2006).

5. Stradner, A. et al. Equilibrium cluster formation in concentrated protein solutions and colloids. Nature 432, 492-495 (2004).

6. Gasser, U., Weeks, E. R., Schofield, A., Pusey, P. N. \& Weitz, D. A. Real-space imaging of nucleation and growth in colloidal crystallization. Science 292, 258-262 (2001).

7. Bibette, J., Mason, T. G., Gang, H. \& Weitz, D. A. Kinetically induced ordering in gelation of emulsions. Phys. Rev. Lett. 69, 981-984 (1992).

8. Clayden, J., Greeves, N., Warren, S. \& Wothers, P. Organic Chemistry 1st edn (Oxford Univ. Press, 2000).

9. Yin, Y. \& Xia, Y. Self-assembly of spherical colloids into helical chains with wellcontrolled handedness. J. Am. Chem. Soc. 125, 2048-2049 (2003).

10. Goubault, C., Leal-Calderon, F., Viovy, J. L. \& Bibette, J. Self-assembled magnetic nanowires made irreversible by polymer bridging. Langmuir 21, 3725-3729 (2005)

11. Manoharan, V. N., Elsesser, M. T. \& Pine, D. J. Dense packing and symmetry in small clusters of microspheres. Science 301, 483-487 (2003).

12. Tandon, S., Beleggia, M., Zhu, Y. \& De Graef, M. On the computation of the demagnetization tensor for uniformly magnetized particles of arbitrary shape. Part I: Analytical approach. J. Magn. Magn. Mater. 271, 27-38 (2004).

13. Young, R. J. \& Lovell, P. A. Introduction to Polymers 2nd edn (Chapman and Hall, 1991).

14. Stöber, W., Fink, A. \& Bohn, E. Controlled growth of monodisperse silica spheres in the micron size range. J. Colloid Interface Sci. 26, 62-66 (1968).

15. Zerrouki, D. et al. Preparation of doublet, triangular, and tetrahedral colloidal clusters by controlled emulsification. Langmuir 22, 57-62 (2006).

Acknowledgements We thank R. Dreyfus for discussions. 